\title{
p-Phenylenediamine and Risk of Sensitization in Children
}

\section{Jakob Ferløv Schwensen, MD, PhD* \\ Jeanne Duus Johansen, MD, DMSC}

\author{
Address \\ *National Allergy Research Centre, University Hospital Gentofte, Niels \\ Andersens Vej 65, 2900, Hellerup, Denmark \\ Email: jakob.ferloev.schwensen.03@regionh.dk
}

Published online: 17 June 2014

(C) Springer International Publishing AG 2014

Keywords $p$-Phenylenediamine (PPD) - Allergic contact dermatitis - Henna - Temporary henna tattoes - Hair dyes

\section{Opinion statement}

Contact allergy to $p$-phenylenediamine (PPD) in children has been described over almost 2 decades, indicating a major problem. PPD can be found in permanent hair dyes and temporary henna tattoos. Children often get sensitized to PPD at a relatively young age when getting a temporary henna tattoo while away on holiday in exotic surroundings. This results in a localized acute allergic reaction at the site of the tattoo within 1-2 weeks after the tattoo has been drawn. The symptoms will clear over weeks on treatment with topical corticosteroids. However, sensitization to PPD will be life-long and may later result in very severe allergic reactions if re-exposure to PPD happens, e.g., by hair dyeing. Populationbased studies indicate that even young children may get their hair dyed and that many teenage girls dye their hair regularly. Exposure to permanent hair dyes with PPD and related substances may in itself lead to PPD sensitization and severe allergic reactions. The allergic reaction will typically develop within hours after dyeing the hair, with clinical presented symptoms such as facial edema and facial dermatitis, which also may generalize. This may lead to hospital admission and treatment with systemic corticosteroids. It is important to note that this is a delayed-type hypersensitivity reaction and should be treated as such. Antihistamines have no effect in this situation. PPD sensitization may have long-term carrier consequences, as, for example, PPD contact allergy in a teenage girl may hinder her entering the hairdressing trade. Patch testing is used to make the diagnosis of PPD sensitization. In children with severe clinical reactions to temporary henna tattoos and/or permanent hair dyes, it is advisable to start the patch testing with PPD in a low concentration, e.g., $0.01 \%$ PPD in petrolatum. It is not recommended to get temporary henna tattoos under any circumstances and children under the age of 16 years should not use permanent hair dyes in order to avoid severe allergic reactions and life-long sensitization to PPD.

\section{Introduction}

Contact allergy has traditionally been considered to occur rarely in the paediatric population, mainly as a result of the lesser exposure to allergens [1-3]. The prevalence of contact allergy in children has over the last decades increased as a result of the more grownup behavior of children (e.g., body piercing, hair dye- 
ing, and use of cosmetics) and more extensive patch testing of children with contact dermatitis [4-6].

p-Phenylenediamine (PPD) is a strong sensitizer known to cause contact allergy in both occupational and domestic settings, e.g., hairdressing or with temporary henna tattooing $[2,7,8 \bullet]$. In children, allergic reactions to PPD have primarily been observed due to temporary henna tattooing and hair dyeing.

\section{Epidemiology, source of exposure, and clinical presentation}

PPD (p-phenylenediamine, paraphenylenediamine, 4-phenylenediamine; CAS Registry Number 106-50-3) is an aromatic amine predominantly used as a precursor in oxidative hair dyes or as a compound in temporary henna tattoos $[8 \bullet, 9,10]$. PPD is a known strong sensitizer and has for centuries been known to cause contact allergy [2, 7, 10-14], which may sensitize by a single or few exposures depending on the exposure concentrations.

Several studies exist concerning contact allergy to PPD in patch-tested children with contact dermatitis [1,3-5]. In a recent Danish study, $3.5 \%$ of all patch tested children had PPD contact allergy [5]. This is in accordance with a Canadian study, which found a prevalence of sensitization to PPD in $4.0 \%$ of patch tested children [4]. In a Danish population-based study of school children aged 12-16 years, $0.2 \%$ had contact allergy to PPD [15]. These articles mainly illustrate the fact that children's exposures may have changed. In the adult population, contact allergy to PPD is associated with female gender and being a hairdresser $[7,10,13,16,17]$. In contrast to this, contact allergy to PPD in children is often observed in both genders [3, 5, $15]$, which may indicate that both genders in young children have an equal risk behavior towards exposure to PPD, e.g., temporary henna tattooing.

\section{Temporary henna tattoos (black henna)}

For centuries, henna has been used to stain hair, skin, and nails for public celebrations and religious ceremonies as an expression of body art. Henna is the dried and powdered leaf of the dwarf evergreen shrub Lawsonia inermis, and when it is applied to skin, the pigment lawsone interacts with keratin, therein producing the rust red color (brown henna) [8• 18].

In recent years, use of henna as body paint refers to black henna used for temporary henna tattooing. Often it is a chemical mixture between the original redbrown henna and substances such as PPD. PPD is added to brown henna to darken and strengthen the color and to accelerate the drying process [8•]. The content of PPD in temporary henna tattoos has been reported to vary between 0.43 and $15.7 \%$, which is higher than the allowed concentration of PPD in permanent hair dyes $[19,20]$. Some preparations of temporary henna tattooing have been observed not to contain brown henna at all [18].

In many families, holidays in exotic surroundings result in temporary henna tattooing in all different kinds of pattern as a holiday souvenir. The temporary henna tattooing may be done at little street shops or market places with limited control of the substances used and with no age limitation. Several case reports concerning children and contact allergy to PPD due to temporary henna tattooing have been published $[19,21-28]$. In a pa- 
tient-based study of consecutive dermatitis patients from Southern Europe, it was found that patients with contact allergy to PPD owing to temporary henna tattoos had a mean age of 14.6 years compared with a mean age of 35 and 46.4 years in cases with contact allergy to PPD owing to permanent hair dyes in occupational and domestic cases, respectively [27]. These results are confirmed by several case reports with children affected by allergic contact dermatitis due to PPD exposure [19, 21-26, 29]. In many of the case reports, the children are as young as 7 years old when they have an allergic reaction to a temporary henna tattoo $[21,22,25,26,28,30,31]$. Typically, a localized dermatitis reaction in the tattoo presenting with erythema, papules, and blistering will develop 8-10 days after the tattoo has been painted for the first time $[21,22,26,28]$. This is a sign of primary sensitization and elicitation has occurred by just one exposure to PPD [21, 22, 25, 26, 28, 30, 31]. More severe systemic reactions have also been observed $[25,26,28]$, and rare symptoms such as generalized erythema multiforme in relation to a temporary henna tattoo have been reported $[32,33]$. It has even been described that temporary henna tattoos often get repainted at the end of the holidays to ensure the decorative henna's appearance for the longest time after returning home [26]. This behavior of repainting temporary henna tattoos is not favorable as the children are at even greater risk of developing sensitization to PPD. Moreover, the localized allergic skin reactions to a temporary henna tattoo may precede more severe systemic reactions to hair dyeing later on in life $[34,35]$.

Hair dyes are widely used and can be divided into three groups: oxidative dyes also called permanent dyes; semi-permanent hair dyes; and temporary dyes [9]. When oxidative coloring is intended, a precursor is needed (e.g., PPD or toluene-2,5-diamine) in the combination with a coupler (e.g., resorcinol) and an oxidizing agent (e.g., $\mathrm{H}_{2} \mathrm{O}_{2}$ ) [9]. PPD is a common used permanent hair dye [11], and hairdressers are at special risk for developing contact allergy to PPD and other permanent hair dyes [7, 13]. The maximum allowed concentration of PPD in permanent hair dyes in the European Union before oxidation is $4 \%$. Furthermore, it is noted on all hair dye products that the product is not intended for use in children under the age of 16 years. This is a recent initiative by the European Union prompted by the many reports of severe reactions to permanent hair dyes in children $[16,31,34-36]$.

It is not known to what extent children and youngsters dye their hair; however, in a Danish study based on a selected material of hairdressing apprentices, mainly girls, and a matched control group of teenagers, it was found that the mean age of their first hair dyeing was 12.1 and 13.3 years, respectively. At the time of response to the questionnaire, the hairdressing apprentices with a mean age of 17.4 years and the controls with a mean age of 17.5 years had dyed their hair 6.6 and 3.7 times within the last year, respectively [37•]. The control group was extracted from the general population and was matched on age, gender, and area code, which may have influenced the data presented. Nevertheless, these data indicate that children, and especially girls, start dyeing their hair at a relatively young age. 
Data on adults from a multicenter study indicate that Middle and Southern European countries have a higher prevalence of contact allergy to PPD than Scandinavia [38]. This could indicate that geographical places with a population with darker skin color and hair, e.g., Southern Europe, could be dyeing their hair more often than, for example, Scandinavians with a higher prevalence of blond hair and different behavior intended to maintain a blonder look [38]. Whether this could be applied to the population of children has not yet been proven.

Allergic skin reactions to PPD vary from localized dermatitis at the scalp and rim of the hair to severe facial and neck edema and generalized rashes with a need for intensive care treatment [34, 35, 39]. Also, dyeing of brows and lashes may cause severe reactions (Fig. 1).

Based on a Danish population-based questionnaire, it was concluded that $5.3 \%$ of adults have had an allergic reaction to dyeing their hair, while only $15.6 \%$ of these had been in contact with healthcare services [16]. No such population-based studies on contact allergy to permanent hair dyes in children exist. The actual number of children with allergic skin reactions is probably underreported $[5,34]$. As previously noted, the change in behavior of children towards more grown-up habits could have influenced on the prevalence of contact allergy to PPD in children due to hair dyeing. Prior sensitization to PPD by temporary henna tattooing due to the high concentrations of PPD in the paste cause a highly sensitized child, who will develop very severe allergic reactions later on if re-exposed to PPD in permanent hair dyes [22, 23, 34, 35].

\section{Cross-reactivity and concomitant reactions}

Cross-reactivity between PPD and a number of substances in the para-aromatic amines have been known for decades [40, 41]. However, little is published regarding cross-reactivity of PPD and other para-aromatic amines in children. Children are often sensitized to PPD due to the afore-mentioned exposures to temporary henna tattooing and/or permanent hair dyes, but they may also have simultaneous positive reactions to other para-aromatic amines when patch tested without ever being exposed to these para-aromatic amine groups. Children with contact allergy to PPD may react to black rubber [34, 42•], other hair dye substances [34], and textile dyes [43, 44]. Seldom, children are sensitized primarily to one of the mentioned para-aromatic amine groups, e.g., black rubber, and then co-react to PPD when patch tested.

The problem with cross-reactivity of a contact allergy to PPD and other para groups could manifest in childhood [44] or later when occupational exposure occurs, e.g., to black rubber in blue-collar workers or other hair dyes in hair-

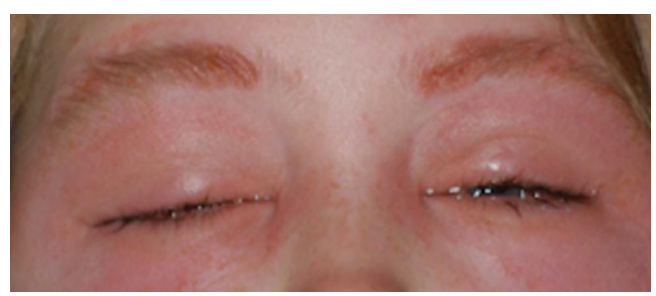

Fig. 1. A 12-year-old girl with periorbital edema and contact dermatitis in the eyebrows following dyeing. Patch test result showed contact allergy to $p$-phenylenediamine. 


\section{Pathogenesis}

dressers. Little is published regarding the later occupational implications for children with contact allergy to PPD. A recent published case report described a junior salesman selling plumbing devices with severe hand dermatitis [42•]. Patch testing revealed that he had contact allergy to several black rubber chemicals, hair dye chemicals, benzocaine, and textile dyes, all known for their cross-reactivity to PPD. The history revealed that he was temporary henna tattooed as a child with eruption of dermatitis at the anatomical site of the temporary henna tattoo [42•]. He had to leave his profession due to periods of sick leave, and this case report shows the potential huge carrier consequence that sensitization to PPD acquired in childhood can have.

Contact allergy, which is a delayed-type (IV) hypersensitivity reaction, is normally induced by repeated allergen exposure to the skin. However, sensitization to PPD may only require one exposure if the concentration is sufficiently high, such as in temporary henna tattooing.

Contact allergy has two phases: the induction and the elicitation phase. The induction phase is the period when the individual is exposed to the allergen (contact sensitizer/hapten) for the first time until the individual is sensitized to the allergen. The elicitation phase is the phase where exposure to the allergen elicits an allergic skin response in an already sensitized individual. The sensitization phase is without symptoms, while clinical skin symptoms are developed in the elicitation phase, typically erythema, edema, and possible vesicles [45].

PPD is a prohapten that is converted to a more reactive hapten; the exact chemical nature of this is still not fully understood [9]. In the induction phase, the hapten penetrates the skin barrier, which activates dendritic cells that migrate to draining lymph nodes with priming of naïve $\mathrm{T}$ cells and later activation of hapten-specific T cells [45-48]. The elicitation phase normally begins with renewed exposure to the hapten, when the hapten is presented to hapten-specific T cells by allergen-presenting cells with the result of local cytokine and chemokine release $[45,49]$. These mediators both have proinflammatory responses and recruit other inflammatory cells, which makes the skin develop acute dermatitis with erythema, edema, infiltration, and vesicles.

When a child gets a temporary henna tattoo, the skin is exposed to PPD for a long period of time (weeks), which means that both the induction and elicitation phase can happen with the one exposure. Several of the aforementioned case reports confirm that only one exposure to PPD, e.g., temporary henna tattooing, is needed to elicit a delayed-type hypersensitivity reaction within days $[21,25,44]$. It is well-known that exposure to PPD in a low concentration and for a short time, e.g., when hair dyeing, can elicit an allergic skin reaction in individuals already sensitized to PPD [50, 51].

For further details, see textbooks or review articles on contact allergy to PPD [9, 50, 52-54].

The diagnosis of allergic contact dermatitis due to PPD is based on a positive patch test result to PPD in a patient with a history of contact dermatitis in relation to exposure to PPD [55]. Patch testing is performed on the upper back of the 


\section{Prevention}

individual using a small aluminium chamber ( $8 \mathrm{~mm}$ ) filled with PPD as free base or as a thin-layer rapid-use epicutaneous patch test. The occlusion time of the patch test chamber is $48 \mathrm{~h}$, and readings are performed at least once at days 3 or 4 and often also at days 2 and 7, in accordance with the recommendations of the International Contact Dermatitis Research Group [56]. Reactions of strength 1+, $2+$, and $3+$ are interpreted as positive responses.

In adults, PPD is included in the European baseline series in a recommend concentration of $1 \%$ in petrolatum.

Children with a previously localized allergic reaction to a temporary henna tattoo and/or severe allergic reaction to permanent hair dyes tend to have strong patch test reactions $(2+/ 3+)[21,22,25,28,30,31,34,35]$ (see Fig. 2). Overall, a trend of strong positive patch test reactions to PPD is shown when the sensitization phase is powered by a temporary henna tattoo with a high concentration of PPD.

In children, patch testing with PPD should be done in case of clinical suspicion of contact allergy to PPD, e.g., in relation to contact dermatitis to temporary henna tattoos or permanent hair dyes, in order to make the diagnosis.

Children should be patch tested with the smallest possible concentration of PPD to avoid strong allergic reactions, as it is known that children with a history of reactivity to PPD due to temporary henna tattooing would need a lower dose and/or less exposure time to elicit an allergic reaction. Therefore, patch testing with PPD is recommended to be performed in a step-wise procedure starting with a low concentration of $0.01 \%$ PPD in petrolatum. If the concentration of $0.01 \%$ PPD has not elicited any allergic skin reaction at the first reading, a new patch test chamber with a stronger concentration can be applied of 0.1 or $1.0 \%$ PPD in petrolatum [57]. This titration of PPD dose ensures that children with a high reactivity to PPD are diagnosed with the lowest possible dose of PPD for the shortest duration of time.

Prevention of contact allergy to PPD should have priority. Children should avoid getting temporary henna tattoos. The listed literature clearly shows that

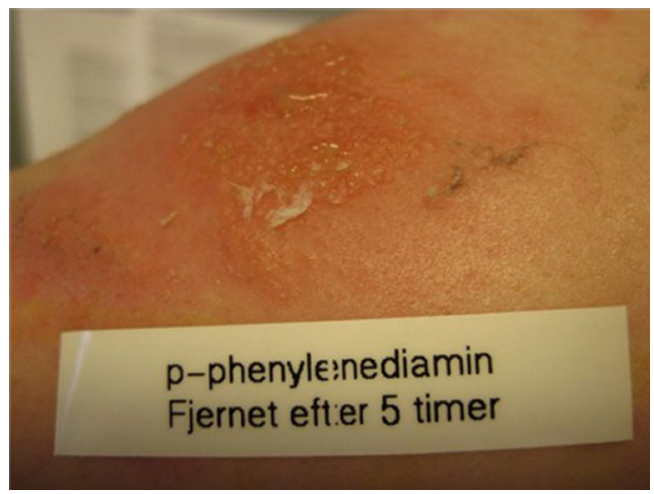

Fig. 2. A strong patch test reaction to $p$-phenylenediamine in $1 \%$ petrolatum. The patch was removed after $5 \mathrm{~h}$ due to a strong allergic reaction. The reading was performed at $48 \mathrm{~h}$. 
only a single episode of getting a temporary henna tattoo is enough to induce contact allergy to PPD, which could have major impact on future carrier choice and the development of severe allergic reactions later in life when re-exposed to PPD or related compounds, e.g., in permanent hair dyes. Children with a history of an allergic reaction following a temporary henna tattoo should therefore avoid hair dyeing and occupational exposures to PPD, e.g., avoiding hairdressing with a daily exposure to hair dyes or black rubber exposure in machine operating and the like. This could have major impact on a young adult's quality of life; e.g., if the dream of becoming a hairdresser is endangered by having a localized skin reaction after getting a temporary henna tattoo as a child.

An age limitation exists on permanent hair dyes, as children under the age of 16 years are recommended not to get their hair dyed with permanent hair dyes. All manufacturers are obliged by law to write this on their products. This recommendation should also be implemented in the hairdressing trade, as many of the case reports show that young teenagers with allergic reactions to PPD had had their hair dyed at the hairdresser [34].

The skin allergy test (SAT) is recommended by the manufacturers as a quick and easy way to test for contact allergy to hair dyes. The SAT is an open application of the coloring base at the retroauricular area for $48 \mathrm{~h}$ prior to hair dyeing. This procedure is not standardized and implies a risk for sensitization to PPD by the procedure itself [17]. Children with a prior allergic skin reaction to a temporary henna tattoo should avoid hair dyeing as they are at risk for developing severe allergic reactions.

\section{Prognosis and treatment}

\section{Conclusions}

Depending on the extent of disease, contact allergy to PPD requires acute management with topical and sometimes systemic corticosteroids. Different national guidelines exist on the use of corticosteroids in children concerning dose and treatment duration.

The use of antihistamines will have no effect on the allergic contact dermatitis, and it is important to distinguish between a delayed-type hypersensitivity reaction and an immediate-type hypersensitivity reaction. The difference between the two hypersensitivity reactions is found in the history, as the immediate-type hypersensitivity reaction often develops over minutes while the delayed-type hypersensitivity reaction takes hours to days before an allergic response is elicited. The acute symptoms will disappear on treatment, but it is of great importance to point out that the sensitization to PPD will persist. Prevention and allergen avoidance is the mainstay to prevent new allergic skin reactions to PPD (see Prevention).

PPD is primarily used in temporary henna tattoos and in permanent hair dyes. Contact allergy to PPD in children has been observed in numerous case reports over the last decade. Children tend to get sensitized to PPD in temporary henna tattoos while away on holidays at places with little or no legislation on the PPD content in these temporary henna tattoos. This 
sensitization to PPD can give localized symptoms such as erythema, papules, and blistering. Often, only one temporary henna tattoo is needed for the child to get sensitized to PPD and to elicit an allergic skin reaction within days.

The literature indicates that children may get their hair dyed at a relatively young age. The exposure to permanent hair dyes with PPD may in itself also lead to PPD sensitization and can elicit severe allergic reactions with facial dermatitis and facial edema within hours of exposure. An allergic reaction due to PPD is a delayed-type hypersensitivity reaction and is treated with either topical or systemic corticosteroids. Antihistamines will have no effect on the allergic reactions. Patch testing is used to make the diagnosis of contact allergy to PPD. In children with severe clinical skin reactions to temporary henna tattoos and/or permanent hair dyes, it is advisable to start the patch testing with PPD in a low concentration, e.g., $0.01 \%$ PPD in petrolatum.

Children are not recommended to get temporary henna tattoos under any circumstances and children under the age of 16 years should not use permanent hair dyes. In already sensitized children, it is important to avoid future occupational and domestic exposure to PPD and other substances known to cause cross-reactivity, e.g., black rubber.

In the future, population-based and patient-based retrospective, epidemiological studies on contact allergy to PPD in children are needed, with a focus on long-term consequences. Moreover, follow-up studies on the effectiveness of the legislation on preventing sensitization to permanent hair dyes in children are warranted, e.g., to investigate the law intended to limit children having their hair dyed when younger than 16 years of age and its implementation in the hairdressing trade.

\section{Compliance with Ethics Guidelines}

\section{Conflict of Interest}

Jakob Ferløv Schwensen and Jeanne Duus Johansen declare that they have no conflict of interest.

Human and Animal Rights and Informed Consent

This article does not contain any studies with human or animal subjects performed by any of the authors.

\section{References and Recommended Reading}

Papers of particular interest, published recently, have been highlighted as:

- Of importance

1. Weston WL, Weston JA. Allergic contact dermati- 3. tis in children. Am J Dis Child. 1984;138 (10):932-6.

2. Marcussen PV. Primary irritant patch-test reactions in children. Arch Dermatol. 1963;87:37882.
Veien NK, Hattel T, Justesen O, Norholm A. Contact dermatitis in children. Contact Dermatitis. 1982;8(6):373-5.

4. Hogeling $M$, Pratt $M$. Allergic contact dermatitis in children: the Ottawa hospital patch-testing clinic experience, 1996 to 2006. Dermatitis: Contact Atopic Occup Drug. 2008;19(2):86-9. 
5. Simonsen AB, Deleuran M, Mortz CG, Johansen JD, Sommerlund M. Allergic contact dermatitis in Danish children referred for patch testing - a nationwide multicentre study. Contact Dermatitis. 2014;70 (2):104-11.

6. Barros MA, Baptista A, Correia TM, Azevedo F. Patch testing in children: a study of 562 schoolchildren. Contact Dermatitis. 1991;25(3):156-9.

7. Frosch PJ, Burrows D, Camarasa JG, DoomsGoossens A, Ducombs G, Lahti A, et al. Allergic reactions to a hairdressers' series: results from 9 European centres. The European Environmental and Contact Dermatitis Research Group (EECDRG). Contact Dermatitis. 1993;28(3):180-3.

8. de Groot AC. Side-effects of henna and semi-permanent 'black henna' tattoos: a full review. Contact Dermatitis. 2013;69(1):1-25.

The article gives a full review on the literature published regarding temporary henna tattooing, contact allergy to PPD, and its consequences for health and later career prospects if children are sensitized to PPD.

9. Basketter D, Johansen JD, McFadden J, Søsted H. Hair dyes. In: Johansen JD, Frosch PJ, Lepoittevin J-P, editors. Contact dermatitis. 5th ed. Springer-Verlag: Berlin; 2011. p. 629-41.

10. Schnuch A, Lessmann H, Frosch PJ, Uter W. paraPhenylenediamine: the profile of an important allergen. Results of the IVDK. Br J Dermatol. 2008;159(2):379-86.

11. Corbett JF. An historical review of the use of hair dye precursors in the formulation of commercial oxidation hair dyes. Dye Pigment. 1999;41(1):127-36.

12. DeLeo VA. p-Phenylenediamine. Dermatitis: Contact Atopic Occup Drug. 2006;17(2):53-5.

13. Schwensen JF, Johansen JD, Veien NK, Funding AT, Avnstorp C, Osterballe M, et al. Occupational contact dermatitis in hairdressers: an analysis of patch test data from the Danish Contact Dermatitis Group, 2002-2011. Contact Dermatitis. 2014;70(4):233-7.

14. Thyssen JP, Carlsen BC, Sosted H, Menne T, Johansen JD. Frequency of p-phenylenediamine sensitization among Danish eczema patients tested between 1985 and 2007. Contact Dermatitis. 2008;59(3):184-5.

15. Mortz CG, Lauritsen JM, Bindslev-Jensen C, Andersen KE. Prevalence of atopic dermatitis, asthma, allergic rhinitis, and hand and contact dermatitis in adolescents. The Odense adolescence cohort study on atopic diseases and dermatitis. Br J Dermatol. 2001;144(3):523-32.

16. Sosted H, Hesse U, Menne T, Andersen KE, Johansen JD. Contact dermatitis to hair dyes in a Danish adult population: an interview-based study. Br J Dermatol. 2005;153(1):132-5.

17. Thyssen JP, White JM, European Society of Contact D. Epidemiological data on consumer allergy to pphenylenediamine. Contact Dermatitis. 2008;59 (6):327-43.
18. Almeida PJ, Borrego L, Pulido-Melian E, GonzalezDiaz O. Quantification of p-phenylenediamine and 2-hydroxy-1,4-naphthoquinone in henna tattoos. Contact Dermatitis. 2012;66(1):33-7.

19. Avnstorp C, Rastogi SC, Menne T. Acute fingertip dermatitis from temporary tattoo and quantitative chemical analysis of the product. Contact Dermatitis. 2002;47(2):119-20.

20. Brancaccio RR, Brown LH, Chang YT, Fogelman JP, Mafong EA, Cohen DE. Identification and quantification of para-phenylenediamine in a temporary black henna tattoo. Am J Contact Dermatitis: Off J Am Contact Dermatitis Soc. 2002;13(1):15-8.

21. Turan H, Okur M, Kaya E, Gun E, Aliagaoglu C. Allergic contact dermatitis to para-phenylenediamine in a tattoo: a case report. Cutan Ocul Toxicol. 2013;32(2):185-7.

22. Le Coz CJ, Lefebvre C, Keller F, Grosshans E. Allergic contact dermatitis caused by skin painting (pseudotattooing) with black henna, a mixture of henna and p-phenylenediamine and its derivatives. Arch Dermatol. 2000;136(12):1515-7.

23. Marcoux D, Couture-Trudel PM, Riboulet-Delmas G, Sasseville D. Sensitization to para-phenylenediamine from a streetside temporary tattoo. Pediatr Dermatol. 2002;19(6):498-502.

24. Akelma AZ, Cizmeci MN, Sarifakioglu E, Mete E. A child with allergic contact dermatitis due to paraphenylenediamine. J Allergy Clin Immunol Pract. 2013;1(4):399-400.

25. Craven JA. A well-circumscribed henna tattoo hypersensitivity reaction. J Paediatr Child Health. 2010;46(7-8):372.

26. Jappe U, Hausen BM, Petzoldt D. Erythemamultiforme-like eruption and depigmentation following allergic contact dermatitis from a paint-on henna tattoo, due to para-phenylenediamine contact hypersensitivity. Contact Dermatitis. 2001;45(4): 249-50.

27. Almeida PJ, Borrego L, Liminana JM. Age-related sensitization to p-phenylenediamine. Contact Dermatitis. 2011;64(3):172-4.

28. Jovanovic DL, Slavkovic-Jovanovic MR. Allergic contact dermatitis from temporary henna tattoo. J Dermatol. 2009;36(1):63-5.

29. Wakelin SH, Creamer D, Rycroft RJ, White IR, McFadden JP. Contact dermatitis from paraphenylenediamine used as a skin paint. Contact Dermatitis. 1998;39(2):92-3.

30. Tan E, Garioch J. Black henna tattoos: coexisting rubber and para-phenylenediamine allergy? Clin Exp Dermatol. 2007;32(6):782-3.

31. Onder M. Temporary holiday "tattoos" may cause lifelong allergic contact dermatitis when henna is mixed with PPD. J Cosmet Dermatol. 2003;2(34):126-30. 
32. Sidwell RU, Francis ND, Basarab T, Morar N. Vesicular erythema multiforme-like reaction to paraphenylenediamine in a henna tattoo. Pediatr Dermatol. 2008;25(2):201-4.

33. Neri I, Giacomini F, Raone B, Patrizi A. Generalized erythema multiforme after localized allergic dermatitis from dark henna tattoo. Pediatr Dermatol. 2009;26(4):496.

34. Sosted H, Johansen JD, Andersen KE, Menne T. Severe allergic hair dye reactions in 8 children. Contact Dermatitis. 2006;54(2):87-91.

35. Jasim ZF, Darling JR, Handley JM. Severe allergic contact dermatitis to paraphenylene diamine in hair dye following sensitization to black henna tattoos. Contact Dermatitis. 2005;52(2):116-7.

36. European Commision, Regulation (EC) No 1223/ 2009 of the European Parliament and of the Council of November 2009 on Cosmetic products. http:// ec.europa.eu/consumers/cosmetics/cosing/ index.cfm?fuseaction=ref_data.regulations. Accessed 14 Apr 2014.

37. Bregnhoj A, Sosted H, Menne T, Johansen JD. Exposures and reactions to allergens among hairdressing apprentices and matched controls. Contact Dermatitis. 2011;64(2):85-9.

This study of hairdressing apprentices and a matched control group from the general population showed a significant problem with youngsters being highly exposed to hair dyes, as they often dyed their hair and started doing so at a relatively young age.

38. Thyssen JP, Andersen KE, Bruze M, Diepgen T, Gimenez-Arnau AM, Goncalo M, et al. pPhenylenediamine sensitization is more prevalent in central and southern European patch test centres than in Scandinavian: results from a multicentre study. Contact Dermatitis. 2009;60(6):314-9.

39. Glatstein MM, Rimon A, Danino D, Scolnik D. Severe allergic contact dermatitis from temporary: "black henna" coloring of the hair during religious cultural celebrations: three different cases, same history. Am J Ther. 2014. doi:10.1097/MJT.0b013e318296f141.

40. Uter W, Lessmann H, Geier J, Becker D, Fuchs T, Richter G, et al. The spectrum of allergic (cross-) sensitivity in clinical patch testing with 'para amino' compounds. Allergy. 2002;57(4):319-22.

41. Mayer RL. Die Überempfindlichkeit gegen Körper von Chinonstruktur. Arch Dermatol Syph.

1928; 156:331-54.

42. Hald M, Menne T, Johansen JD, Zachariae C. Severe occupational contact dermatitis caused by black rubber as a consequence of p-phenylenediamine allergy resulting from a temporary henna tattoo. Contact Dermatitis. 2013;68(6):377-9.

This case report of a young man working as junior sales man selling plumbing devices shows the potential huge carrier consequence that sensitization to PPD acquired in childhood can have.
43. Boschnakow A, Treudler R, Lieps D, Steinhoff M, Orfanos CE. Temporary tattooing with henna induces contact allergy to textile dyes [in German]. Journal der Deutschen Dermatologischen Gesellschaft = Journal of the German Society of Dermatology : JDDG. 2003;1(12):962-4.

44. Kiec-Swierczynska M, Krecisz B, SwierczynskaMachura D. Allergy to p-phenylenediamine from a black transferable picture tattoo - hypopigmentation and sensitization to clothing dyes in a little girl. Contact Dermatitis. 2008;58(3):174-5.

45. Rustemeyer T, van Hoogstraten IMW, von Blomberg BME, Gibbs S, Scheper RJ. Mechanisms of irritants and allergic contact dermatitis. In: Johansen JD, Frosch PJ, Lepoittevin J-P, editors. Contact dermatitis. 5th ed. Berlin: Springer-Verlag; 2011. p. 43-90.

46. Macatonia SE, Knight SC, Edwards AJ, Griffiths S, Fryer P. Localization of antigen on lymph node dendritic cells after exposure to the contact sensitizer fluorescein isothiocyanate. Functional and morphological studies. J Exp Med. 1987;166(6):1654-67.

47. Saeki H, Moore AM, Brown MJ, Hwang ST. Cutting edge: secondary lymphoid-tissue chemokine (SLC) and CC chemokine receptor 7 (CCR7) participate in the emigration pathway of mature dendritic cells from the skin to regional lymph nodes. J Immunol. 1999;162(5):2472-5.

48. Steinman R, Hoffman L, Pope M. Maturation and migration of cutaneous dendritic cells. J Invest Dermatol. 1995;105(1 Suppl):2S-7S.

49. Szczepanik M, Akahira-Azuma M, Bryniarski K, Tsuji RF, Kawikova I, Ptak W, et al. B-1 B cells mediate required early $\mathrm{T}$ cell recruitment to elicit protein-induced delayed-type hypersensitivity. J Immunol. 2003;171(11):6225-35.

50. McFadden JP, Yeo L, White JL. Clinical and experimental aspects of allergic contact dermatitis to paraphenylenediamine. Clin Dermatol. 2011;29(3):316-24.

51. Hextall JM, Alagaratnam NJ, Glendinning AK, Holloway DB, Blaikie L, Basketter DA, et al. Dosetime relationships for elicitation of contact allergy to para-phenylenediamine. Contact Dermatitis. 2002;47(2):96-9.

52. Rustemeyer T, van Hoogstraten IMW, von Blomberg BME, Gibbs S, Scheper RJ. Mechanisms of irritant and allergic contact dermatitis. In: Johansen JD, Frosch PJ, Lepoittevin J-P, editors. Contact dermatitis. 5th ed. Berlin: Springer-Verlag; 2011. p. 43-91.

53. Pot LM, Scheitza SM, Coenraads PJ, Blomeke B. Penetration and haptenation of $\mathrm{p}$ phenylenediamine. Contact Dermatitis. 2013;68 (4):193-207.

54. White JM, Kullavanijaya P, Duangdeeden I, Zazzeroni R, Gilmour NJ, Basketter DA, et al. pPhenylenediamine allergy: the role of Bandrowski's base. Clin Exp Allergy: J Br Soc Allergy Clin Immunol. 2006;36(10):1289-93. 
55. Menne T, Johansen JD, Sommerlund M, Veien NK, Danish Contact Dermatitis G. Hand eczema guidelines based on the Danish guidelines for the diagnosis and treatment of hand eczema. Contact Dermatitis. 2011;65(1):3-12.

56. Wilkinson DS, Fregert $\mathrm{S}$, Magnusson B, Bandmann HJ, Calnan CD, Cronin E, et al. Terminology of con- tact dermatitis. Acta Derm Venereol. 1970;50

(4):287-92.

57. Ho SG, White IR, Rycroft RJ, McFadden JP. A new approach to patch testing patients with paraphenylenediamine allergy secondary to temporary black henna tattoos. Contact Dermatitis. 2004;51 (4):213-4. 